\title{
Considérations sur la cinétique chimique de l'échauffement du lait à ultra-haute température
}

par

\author{
J. MOTTAR*
}

\section{INTRODUCTION}

$\mathrm{Au}$ cours du traitement thermique du lait, certains composants peuvent subir des modifications indésirables, nuisibles à la qualité organoleptique et nutritionnelle. D'autre part, pour que le lait devienne conservable, il faut lui appliquer une charge thermique suffisamment élevée pour détruire les micro-organismes et les enzymes.

En introduisant l'échauffement à ultra-haute température, on a tenté d'obtenir un produit conservable, présentant de meilleures caractéristiques de qualité que le lait stérilisé par la méthode traditionnelle. La soumission du lait à un traitement UHT indirect implique cependant le danger que l'échauffement soit si intense que la qualité du lait en sera influencée défavorablement et que l'identité du lait UHT risquera de se perdre [9.].

A la lumière de considérations de cinétique chimique, nous avons tenté de donner une description mathématique d'un certain nombre de réactions, désirables ou indésirables, qui se produisent au cours de l'échauffement à ultra-haute température du lait. De ces données nous avons déduit des critères permettant de déterminer les combinaisons de température et de durée dans la zone UHT auxquelles on obtient un produit conservable, tout en restant d'une qualité acceptable, suffisamment différente de celle du lait stérilisé dans la bouteille.

* Station Laitière de l'Etat, Melle, Centre de Recherches Agronomiques de l'Etat, Gand (Belgique). 


\section{MATERIEL ET METHODES}

\section{II.1. Conditions de traitement}

Du lait entier cru a été porté de façon indirecte à ultra-haute température dans un appareil UHT pilote (APV), équipé d'un échangeur de chaleur à plaques dans sa section UHT. Le système ne comportait pas de récupération de chaleur. Le lait était préchauffé à $80^{\circ} \mathrm{C}$, puis homogénéisé en deux étapes sous des pressions de respectivement 21,6 et 4,9 MPa. Une tuyauterie appropriée, montée en aval du dispositif de chauffage à UHT permettait de régler le temps de séjour moyen dans les limites désirées. Les ultra-hautes températures, de $130^{\circ} \mathrm{C}$ à $150^{\circ} \mathrm{C}$, étaient contrôlées par des thermocouples. Chaque combinaison de température et de durée a été répétée quatre fois, de sorte que les résultats reproduits sont les moyennes de quatre observations.

Les temps de séjour indiqués sont des moyennes et on admet que les effets de la distribution des temps de séjour peuvent être négligés.

Les essais ont été effectués avec un lait de mélange, comme on en traite dans la pratique et dont le nombre de germes se situait entre $2.10^{5}$ et $5.10^{5} / \mathrm{ml}$. Les paramètres de cinétique des réactions doivent donc être considérés comme des moyennes.

\section{II.2. Détermination de l'effet sporicide}

L'efficacité stérilisatrice (ES) a été déterminée selon la méthode de Galesloot [6] et calculée selon la formule :

$$
\mathrm{ES}=\log \frac{\text { nombre de spores avant stérilisation }}{\text { nombre de spores après stérilisation }}=\log \frac{\mathrm{N}_{\circ}}{\mathrm{N}}
$$

Les spores de la souche thermorésistante Bacillus subtilis 0125 (NIZO) ont été choisies comme organisme de référence. Les suspensions de culture sont obtenues en ensemençant un milieu liquide, tel que décrit par Williams et al. [13], d'une culture de base. Après $24 \mathrm{~h}$ d'incubation à $37^{\circ} \mathrm{C}$, la suspension de bactéries multipliées a été incubée en flacons de Roux, dans un milieu au manganèse [3]. Après $7 \mathrm{j}$ d'incubation à $37^{\circ} \mathrm{C}$, les colonies ont été rincées au moyen de $10 \mathrm{ml}$ de solution de Ringer et lavées deux fois. La suspension a été chauffée ensuite à $80^{\circ} \mathrm{C}$ pendant $10 \mathrm{~min}$.

Nous avions veillé à avoir dans le lait, avant la stérilisation, un taux de spores initial ( $\mathrm{N}_{\circ}$ ) d'au moins $10^{7} / \mathrm{ml}$. La teneur en spores a été déterminée par repiquage sur Plate Count Agar, après échauffement du lait à $80^{\circ} \mathrm{C}$ pendant $10 \mathrm{~min}$. La différence entre les nombres de germes/ml avant $\left(\mathrm{N}_{\mathrm{o}}\right)$ et après $(\mathrm{N})$ le traitement UHT a permis de calculer l'effet sporicide. 


\section{II.3. Méthodes analytiques}

Les fractions azotées ont été obtenues par la technique d'Aschaffenburg et Drewry [1]. L'azote a été dosé par micro-Kjeldahl. La teneur en protéines lactosériques (WPN) a été calculée d'après la différence entre l'azote non caséinique (NCN) et l'azote non protéique (NPN). Partant du taux restant de WPN du lait UHT, on pouvait calculer le degré de dénaturation des protéines lactosériques et l'exprimer en p. 100 par rapport à la teneur en protéines lactosériques du lait de départ.

La détermination de la teneur en hydroxyméthylfurfural total (HMF) était basée sur la méthode de Keeney et Basette [7.]. La concentration de l'HMF total a été déduite de l'équation de régression $\mathrm{Y}=87,5 \mathrm{X}-4,8(\mathrm{X}=$ densité optique mesurée à $443 \mathrm{~nm})$.

La lysine disponible a été déterminée par la méthode modifiée de Carpenter, telle que décrite par Booth [2].

L'activité des protéases du lait cru et du lait UHT a été mesurée par incubation anaérobique de ces laits à $37^{\circ}$ C. 0,01 p. 100 de thimérosal a été ajouté au lait, comme agent bactériostatique [11]. L'azote non caséique a été déterminé après $0,3,6,9$ et 12 j. L'activité protéolytique a été calculée par analyse de régression linéaire simple et exprimée comme augmentation de $\mathrm{mg}$ de NCN par $100 \mathrm{~g}$ de lait par 24 h. L'activité résiduelle dans le lait UHT a été exprimée en p. 100 par rapport à l'activité dans le lait de départ.

\section{CINETIQUE DE LA DESTRUCTION DES SPORES ET DES MODIFICATIONS CHIMIQUES}

La mesure dans laquelle la destruction des spores de bactéries et les modifications chimiques dépendent de la température peut être définie par des paramètres de cinétiques chimiques. La valeur $\mathrm{z}$, c'est-à-dire la valeur de résistance à la chaleur, fournit d'importantes informations sous ce rapport. Cette valeur est définie comme l'augmentation de température $\left({ }^{\circ} \mathrm{C}\right)$ nécessaire pour obtenir en un temps d'échauffement 10 fois plus court le même effet sporicide ou le même déroulement d'une réaction [8].

La valeur $z$ est en relation avec le coefficient de température $\mathrm{Q}_{10}$ [8]. La valeur $\mathrm{Q}_{10}$ indique le facteur d'accélération ou de ralentissement d'une réaction en cas d'élévation ou de baisse de $10^{\circ} \mathrm{C}$ de la température :

$$
\log \mathrm{Q}_{10}=\frac{10}{\mathrm{z}}[1]
$$

Il existe également un rapport entre la valeur $\mathrm{z}$ et l'énergie d'activation Ea [8]. Il est exprimé par l'équation d'Arrhenius, qui indique 
la relation entre la vitesse de déroulement d'une réaction et la température :

$$
\mathrm{k}=\mathrm{A} \cdot \mathrm{e}-\mathrm{Ea} / \mathrm{RT}[2]
$$

où $\mathrm{k}=$ la constante de vitesse de réaction $\left(\mathrm{s}^{-1}\right)$.

$A=$ constante $\left(\mathrm{s}^{-1}\right)$.

$\mathrm{Ea}=$ l'énergie d'activation $\left(\mathrm{k} J \cdot \mathrm{mol}^{-1}\right)$.

$\mathrm{R}=$ la constante des gaz $\left(8,31 \mathrm{k} \mathrm{J} \cdot \mathrm{mol}^{-1} \cdot \mathrm{K}^{-1}\right)$.

$\mathrm{T}=$ la température absolue $(\mathrm{K})$.

On peut déduire de l'équation [2] que la différence entre deux vitesses de réaction $k_{1}$ et $k_{2}$, correspondant aux températures $T_{1}$ et $\mathrm{T}_{2}$, s'exprime par l'équation :

$$
\log \mathrm{k}_{2}-\log \mathrm{k}_{1}=\frac{\mathrm{E}_{\mathrm{a}}}{2,3 \mathrm{R} \cdot \mathrm{T}_{1} \mathrm{~T}_{2}}\left(\mathrm{~T}_{1}-\mathrm{T}_{2}\right)[3]
$$

En outre, on a, par définition :

$$
\mathrm{Q}_{10}=\frac{\mathrm{k}_{\mathrm{T}}+10}{\mathrm{k}_{\mathrm{T}}}[4]
$$

Dès lors on peut déduire des équations [1,2] et [3] que :

$$
\mathrm{z}=\frac{2,3 \cdot \mathrm{R} \cdot \mathrm{T}_{1} \cdot \mathrm{T}_{2}}{\mathrm{E}_{\mathrm{a}}}[5]
$$

L'équation [5] fait apparaître la dépendance de la valeur z par rapport à la température, ainsi que l'influence de l'énergie d'activation.

Ces paramètres de cinétiques chimiques permettent d'apprécier les effets bactériologiques et chimiques d'un processus d'échauffement. Pour des intervalles de température assez étroits, on peut admettre que la relation log temps de séjour/température est linéaire [8].

\section{RESULTATS ET COMMENTAIRES}

\section{IV.1. Cinétique de destruction des spores et des modifications chimiques lors de l'échauffement à ultra-haute température du lait}

\section{IV.1.1. Destruction DES SPORES THERMO-RÉSISTANTES}

Les courbes de destruction de Bacillus subtilis 0125 , dans la zone de température $130^{\circ} \mathrm{C}-150^{\circ} \mathrm{C}$, sont reproduites dans la figure 1 . La destruction des spores est linéaire dans un diagramme semi-logarithmique de température et de temps. On peut déduire de l'inclinaison des droites que la valeur de résistance à la chaleur, ou valeur $\mathrm{z}$, est de $10,7^{\circ} \mathrm{C}$. Ceci permet de calculer une valeur $\mathrm{Q}_{10}$ de 8,60 et une énergie d'activation $\mathrm{E}_{\mathrm{a}}$ de $305 \mathrm{k} \mathrm{J} \cdot \mathrm{mol}^{-1}$. 
L'efficacité sporicide requise d'un traitement thermique est en relation avec le nombre de spores thermorésistantes présentes dans le lait cru, avec le degré de résistance à la chaleur de ces spores et avec le nombre de spores thermorésistantes toléré dans le produit final. Une perte de produit ne dépassant pas 0,01 p. 100 est commercialement acceptable [5]. Le lait cru contient en hiver plus de spores résistantes à la chaleur qu'en été, mais leur nombre serait généralement inférieur à 10 au $\mathrm{ml}$ [12]. Si l'on se base sur une contamination maximale de 10 spores par $\mathrm{ml}$ de lait cru, on peut conclure qu'un effet de stérilisation d'au moins 8 est indispensable. Le développement ultérieur de spores dans le lait UHT est inhibé par des composants chimiques formés du fait de l'échauffement [5]. Ceci réduit encore plus la perte de produit.

\section{IV.1.2. DÉnATURATION DES PROTÉINES LACTOSÉRIQUES}

Les protéines lactosériques sont susceptibles de dénaturation sous l'effet de la chaleur. La détermination du degré de dénaturation fournit des indications quant à l'intensité d'un traitement thermique.

La dénaturation des protéines lactosériques en fonction de la température et de la durée du traitement UHT est reproduite dans la figure 2. Si on relie les points qui correspondent à un même degré de dénaturation, on obtient des droites, dont on peut déduire que la valeur $\mathrm{z}$ est égale à $28,6^{\circ} \mathrm{C}$. Partant de ce résultat, le calcul donne une valeur $\mathrm{Q}_{10}$ de 2,23. Pour la zone de températures considérée, l'énergie d'activation entraînant la dénaturation des protéines lactosériques est de $117 \mathrm{k} \mathrm{J} \cdot \mathrm{mol}^{-1}$.

\section{IV.1.3. FORMATION D'HYDROXYMÉTHYLFURFURAL}

L'hydroxyméthylfurfural (HMF) est un produit intermédiaire de la réaction de Maillard. Sa formation dépend étroitement de la température. La teneur en HMF peut servir de mesure de la réaction de Maillard [4].

La figure 3 montre la formation d'HMF résultant de différentes combinaisons de température et de temps. Les résultats montrent qu'elle peut être figurée par des droites dans un diagramme de logarithme temps-température. La valeur $\mathrm{z}$ déterminant la formation d'HMF est de $27,8^{\circ} \mathrm{C}$, le coefficient de température de 2,29 et l'énergie d'activation de $119 \mathrm{k} \mathrm{J} \cdot \mathrm{mol}^{-1}$.

\section{IV.1.4. Pertes de Lysine disponible}

La lysine (acide $\alpha, \varepsilon$-diaminocaproïque) est un acide aminé essentiel qui doit être fourni entièrement par la nourriture. Le lait en est une excellente source. Sous l'effet de l'échauffement, la lysine peut tou- 


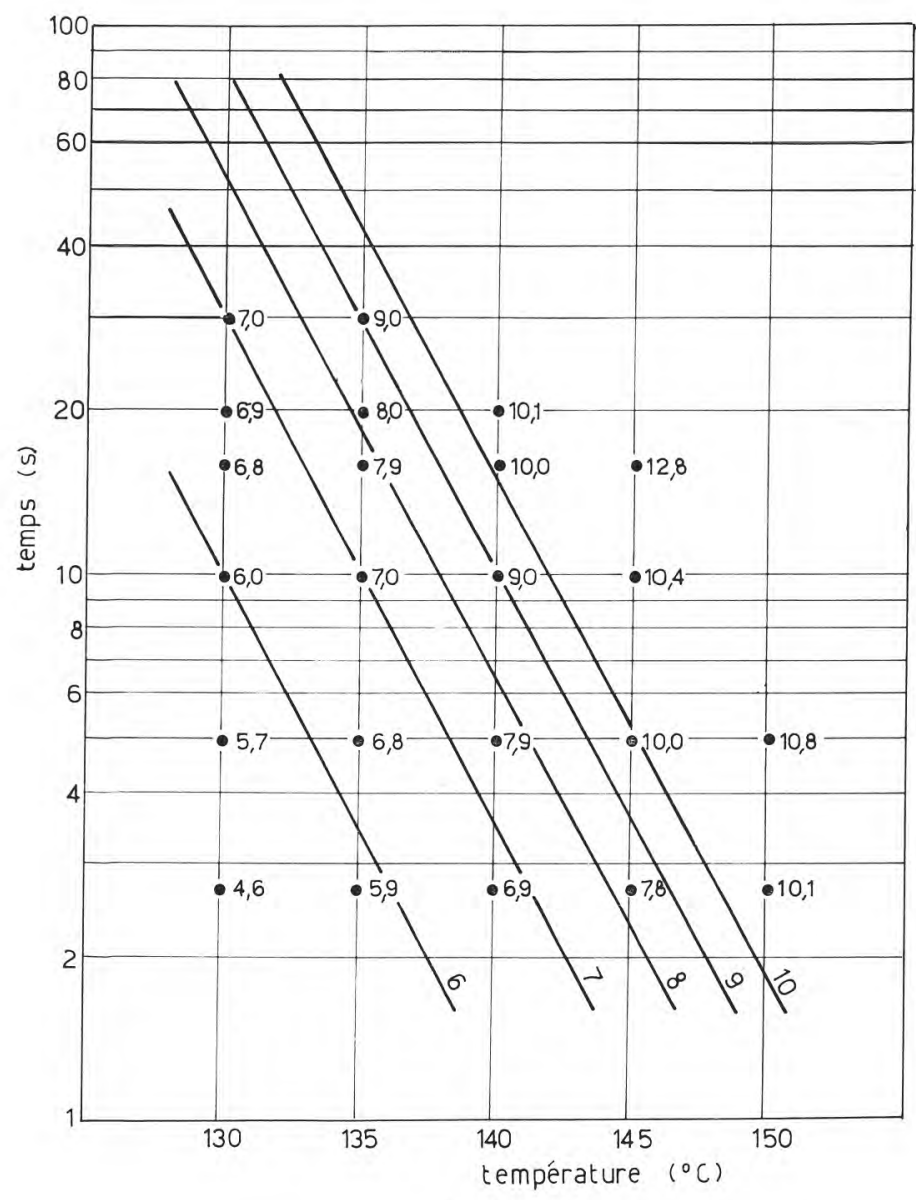

fig. 1

Destruction des spores B. subtilis 0125 en fonction de la température et de la durée d'un traitement UHT indirect.

tefois être impliquée dans la réaction de Maillard, ce qui entraîne la perte de sa valeur nutritionnelle.

Les pertes de lysine mesurées pour différentes combinaisons de température et de temps, sont reproduites dans la figure 4 . On peut en déduire des limites linéaires d'effet égal, dans un diagramme semilogarithmique. Les droites permettent de conclure, par extrapolation, à une valeur de résistance thermique de $30,2^{\circ} \mathrm{C}$. Le coefficient de température $\mathrm{Q}_{10}$ est de 2,14 et l'énergie d'activation de $108 \mathrm{k} \mathrm{J} \cdot \mathrm{mol}^{-1}$, pour l'intervalle de température entre $130^{\circ} \mathrm{C}$ et $150^{\circ} \mathrm{C}$. 


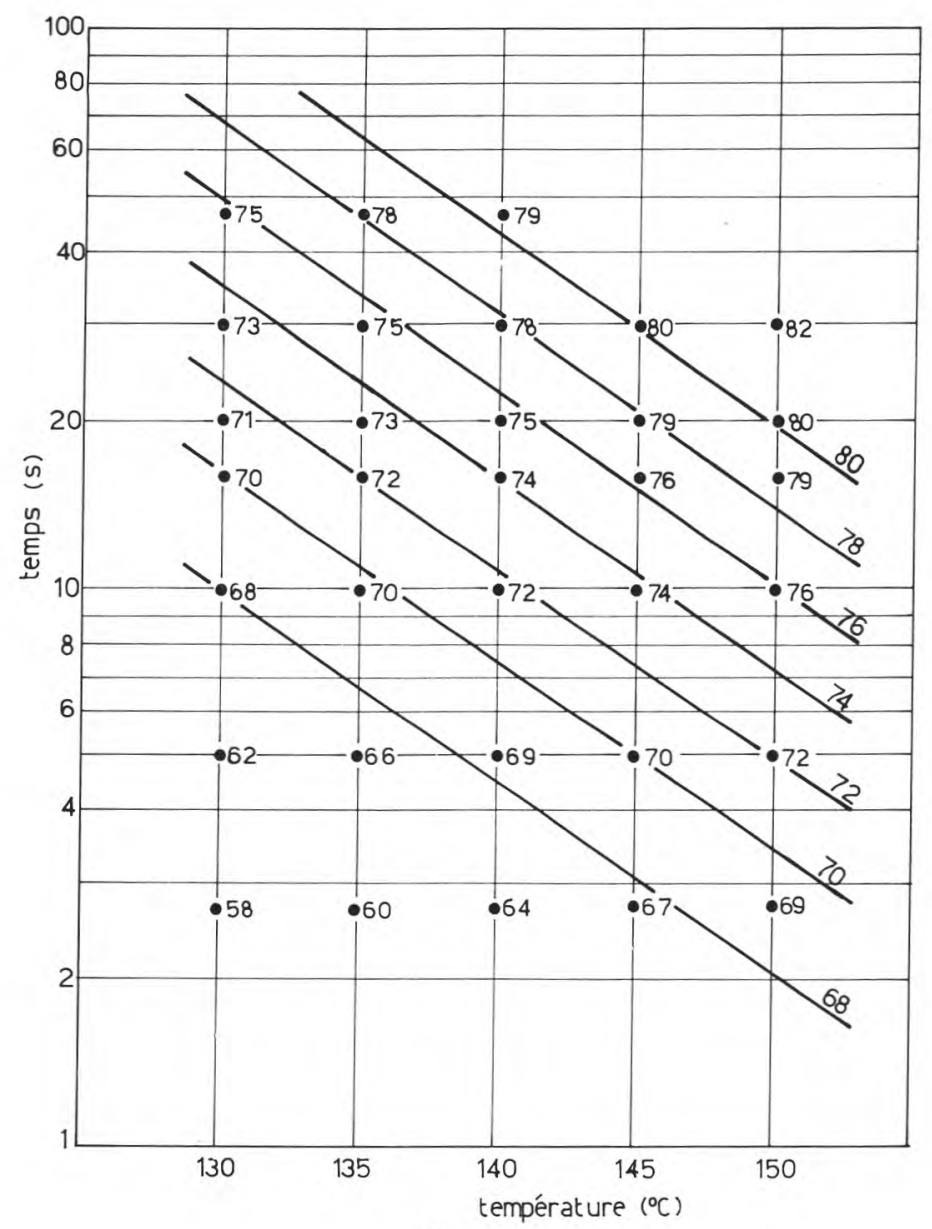

fig. 2

Dénaturation des protéines lactosériques (p. 100) en fonction de la température et de la durée d'un traitement UHT indirect.

\section{IV.1.5. INACTIVATION DES ENZYMES PROTÉOLYTIQUES}

La détérioration du lait UHT pendant la conservation peut être imputée principalement à l'action de protéases [10]. Ce sont donc ces enzymes que le traitement UHT doit inactiver en très grande partie. La figure 5 reproduit les résultats d'inactivation d'enzymes protéolytiques, obtenus par des ultra-hautes températures et différentes durées d'exposition. Les points d'égale inactivation sont à relier par des droites lorsque le temps d'exposition est reporté à l'échelle loga- 


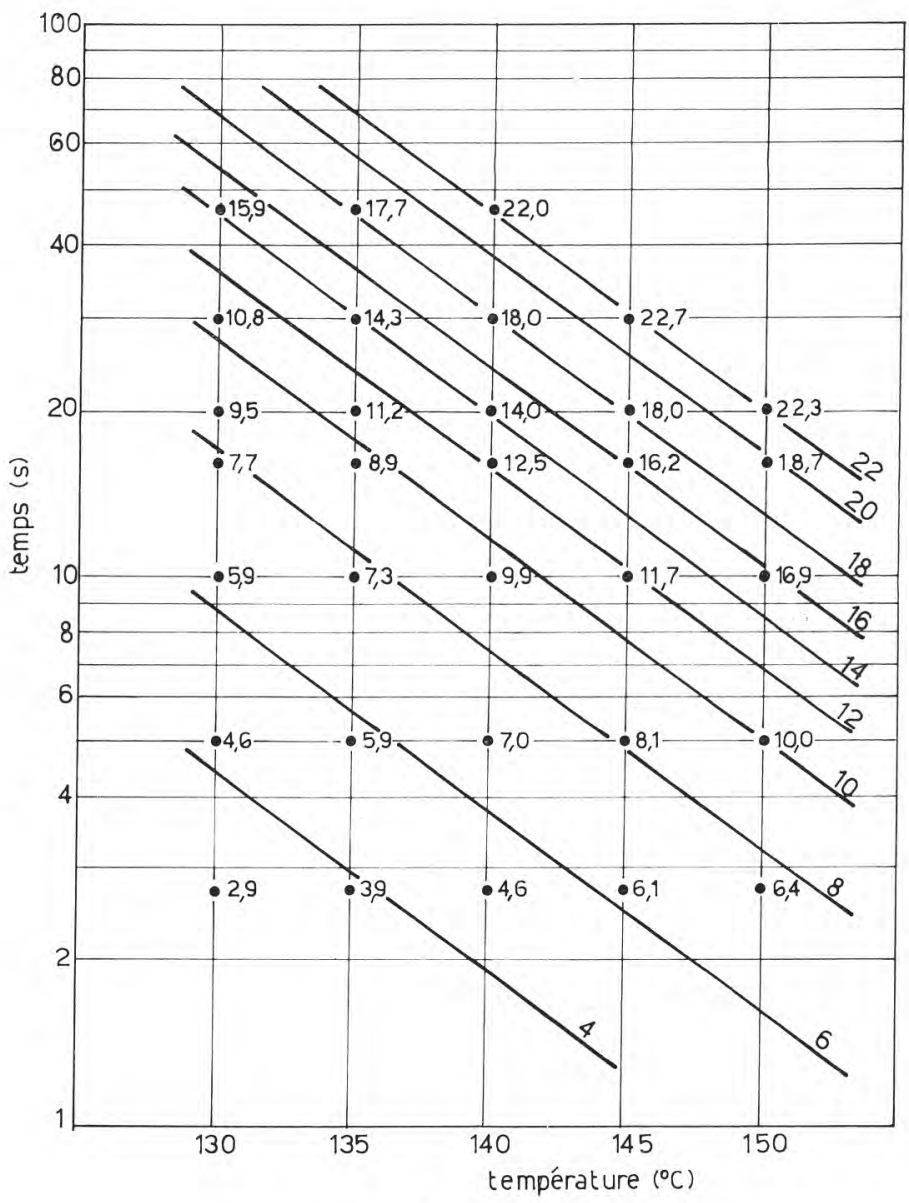

fig. 3

Formation d'hydroxyméthylfurfural $(\mu \mathrm{mol} / \mathrm{l})$ en fonction de la température et de la durée d'un traitement UHT indirect.

rithmique en regard de la température. On peut déduire de l'inclinaison des droites une valeur de $\mathrm{z}$ de $24,9^{\circ} \mathrm{C}$, avec un coefficient de température de 2,52 et une énergie d'activation de $131 \mathrm{k} \mathrm{J} \cdot \mathrm{mol}^{-1}$.

\section{IV.2. Limitation de la zone UHT dans le procédé à échauffement indirect}

Une limitation des températures ultra-hautes et des temps d'exposition correspondants est importante pour la qualité et l'identité du lait UHT, obtenu par la méthode d'échauffement indirect. 


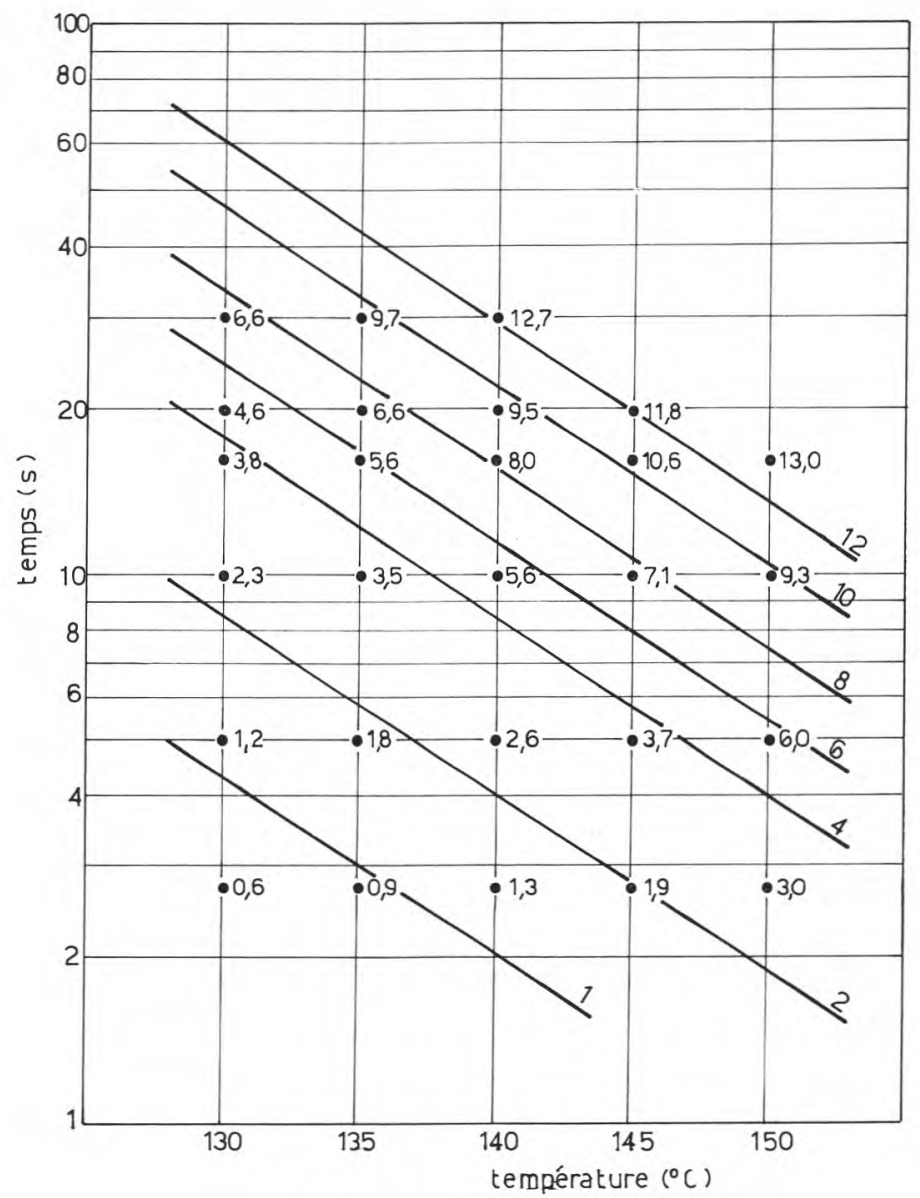

fig. 4

Pertes de lysine disponible (p. 100) en fonction de la température et de la durée d'un traitement UHT indirect.

Il ressort d'une étude comparative, faite par Mottar et Naudts [9], que dans les procédés UHT indirects la charge thermique doit être maintenue dans certaines limites si l'on veut que les caractéristiques du lait UHT restent distinctes de celles du lait stérilisé en deux étapes. La dénaturation des proteines lactosériques du lait UHT, par exemple, doit rester inférieure à 72 p. 100, la teneur en HMF doit rester en dessous de $10 \mu \mathrm{mol} / 1$ et les pertes de lysine disponible ne peuvent pas atteindre 6 p. 100 . En regard de ces limitations des modifications chimiques, la destruction des spores et des enzymes protéo- 


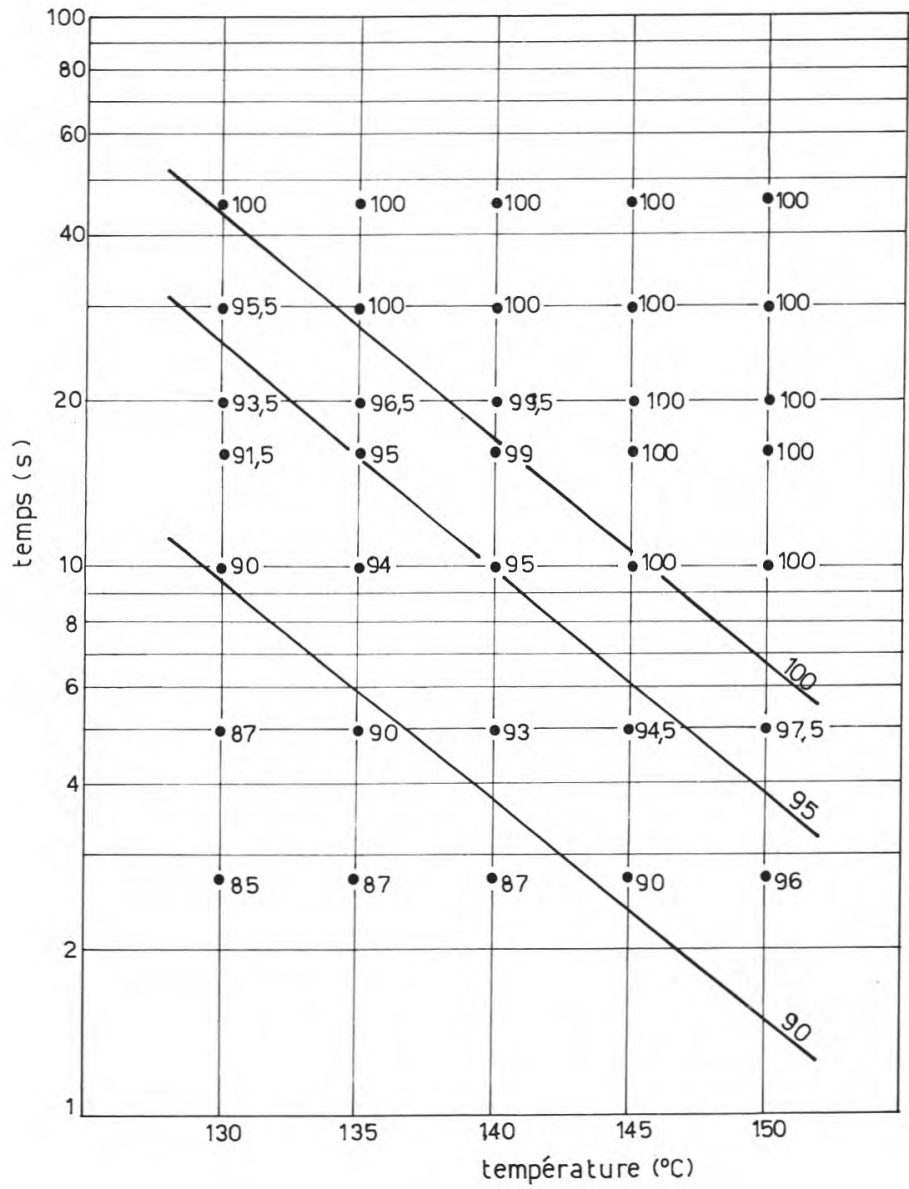

fig. 5

Inactivation de protéases (p.100) en fonction de la température et de la durée d'un traitement UHT indirect.

lytiques doit, par contre, être suffisante pour que le produit final soit conservable.

Compte tenu de ces exigences, les données obtenues permettent de circonscrire une zone UHT dans le diagramme des températures et des temps, comme le montre la figure 6. Dans la zone de tempéra tures d'échauffement et de durées d'exposition, qui s'y trouve indiquée, on peut préparer par le procédé d'échauffement indirect un lait UHT qui garde son identité et est suffisamment conservable. Pour de tels traitements UHT on obtient une turbidité visuellement obser- 


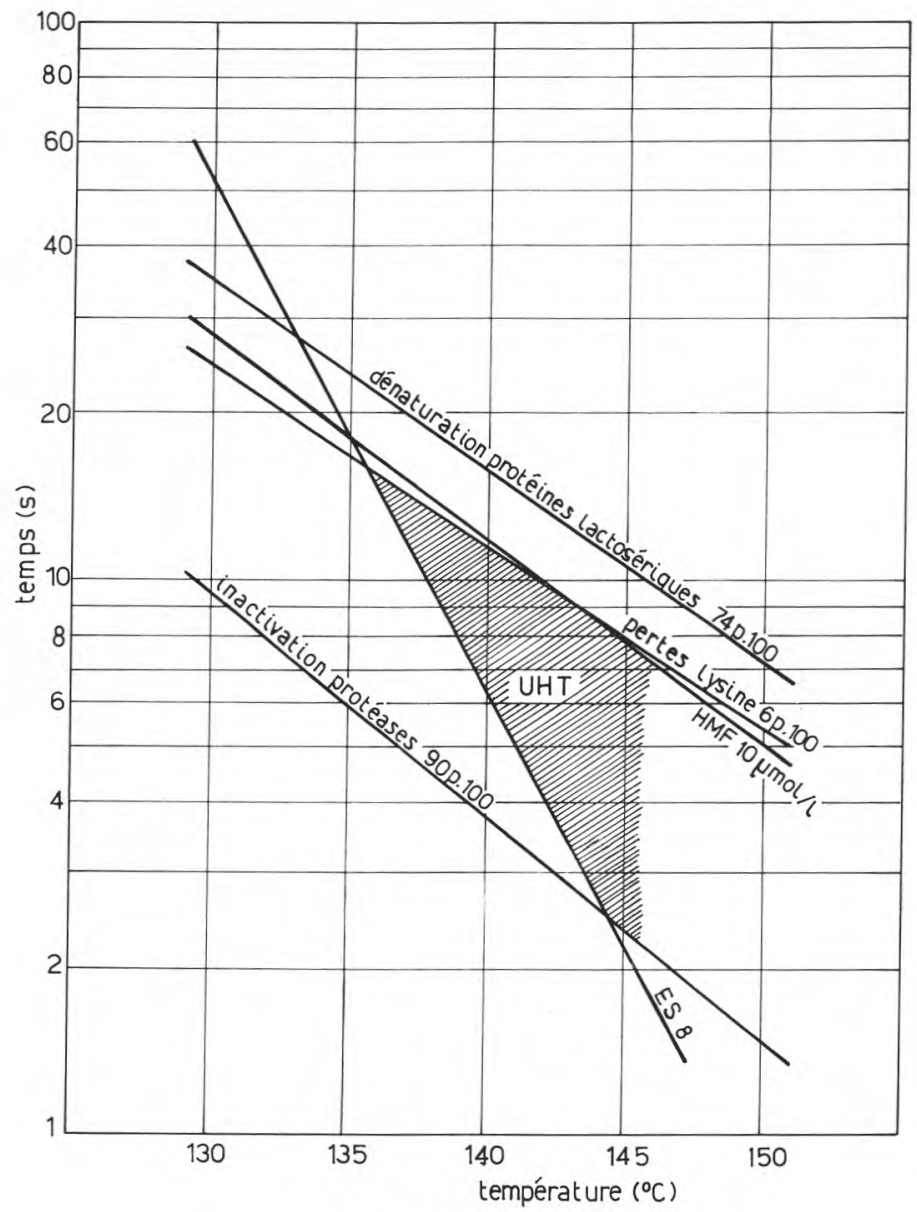

fig. 6

Combinaisons limites de température et de durée d'exposition préservant le caractère UHT du lait.

vable quand on soumet le lait à l'épreuve de turbidité. Pour une température de référence de $142^{\circ} \mathrm{C}$ on peut déduire que le temps d'exposition sera d'au moins $4 \mathrm{~s}$ et de tout au plus $10 \mathrm{~s}$.

A remarquer que les essais auxquels il est référé ici ont été effectués dans une installation pilote, sans récupération de chaleur. Ceci signifie que les températures et durées d'exposition admissibles dans un traitement UHT diminueront à mesure que la récupération de chaleur augmente. 


\section{v. CONCLUSION}

Pour des processus dépendant de la température, tels que la destruction des spores, la dénaturation des protéines lactosériques, la formation d'HMF, les pertes de lysine disponible et l'inactivation d'enzymes protéolytiques, dans la zone des ultra-hautes températures $\left(130^{\circ} \mathrm{C}-150^{\circ} \mathrm{C}\right)$, les effets d'action égale peuvent être représentés par les droites dans un système semi-logarithmique de coordonnées de temps et de température.

Les paramètres de cinétique chimique $\mathrm{z}, \mathrm{Q}_{10}$ et $\mathrm{E}_{1}$, peuvent être calculés d'après l'inclinaison de ces droites. D'après les données ainsi obtenues et moyennant l'introduction de limites au déroulement d'une réaction déterminée, on peut circonscrire une zone UHT dans un diagramme de température et de temps. Les températures et durées d'exposition situées dans la zone indiquée, garantissent une conservabilité suffisante et la préservation d'une qualité acceptable du lait UHT fabriqué par la méthode d'échauffement indirect.

\section{Résumé}

L'influence exercée sur le lait entier par l'échauffement indirect à différentes ultra-hautes températures a été étudiée et caractérisée par des paramètres de cinétique chimique. L'attention s'est portée sur la destruction de spores thermo-résistantes (Bacillus subtilis, 0125) la dénaturation des protéines sériques, la réaction de Maillard (formation d'HMF), les pertes de lysine disponible et l'inactivation des enzymes protéolytiques.

Pour les différentes réactions, on a obtenu dans un diagramme de logarithmes de temps et de température des droites représentant les effets d'action égale. L'inclinaison de ces droites a permis de calculer les grandeurs cinétiques $\mathrm{z}, \mathrm{Q}_{10}$ et $\mathrm{E}_{\mathrm{a}}$.

En manipulant certaines valeurs limites relatives à l'efficacité sporicide le degré de dénaturation des protéines lactosériques, la formation d'HMF, la perte de lysine disponible et l'inactivation des protéases, on a pu circonscrire une zone UHT dans les systèmes des coordonnées de température et de temps. Les échauffements indirects à UHT situés dans cette zone donnent un produit qui, d'une part, est suffisamment conservable, mais dans lequel, d'autre part, les modifications chimiques restent dans des limites acceptables, de sorte que l'identité du lait UHT ne peut être mise en question. 


\section{S u m m a ry}

\section{REACTION KINETIC CONSIDERATIONS ON THE ULTRA-HIGH TEMPERATURE TREATMENT OF MILK}

The influence of various indirect UHT treatments on whole milk was investigated and characterized by reaction kinetic parameters. Attention was paid to the destruction of heat resistant spores (Bacillus subtilis 0125), the heating intensity (whey protein denaturation), the Maillard reaction (HMF formation), the losses in available lysine and the inactivation of proteolytic enzymes.

The log-time/temperature diagrams drawn for the various reactions showed straight lines for effects of similar activities. The slope of the straight lines permitted calculation of the reaction kinetic parameters $\mathrm{z}, \mathrm{Q}_{10}$ and $\mathrm{E}_{\mathrm{a}}$.

The application of particular boundary values to the sterilizing effect, the whey protein denaturation level, the HMF formation, the loss in available lysine and the inactivation of proteases permitted the limitation of a UHT area within the temperature/time coordinate system.

Indirect UHT treatments in this area yield milk whose shelf life is satisfactory and whose chemical changes lie within reasonable limits, so that the identity of UHT milk is not affected.

Reçu pour publication en mars 1981.

\section{Bibliographie}

[1] AschafFenburg (R.) and Drewry (J.) (1959). - New procedure for the routine determination of the various non-casein proteins of milk. 15th Int. Dairy Congress, 3 (5), 1631.

[2] Воотн (V.) (1971). - Determination of FDNB-available lysine. A modified Carpenter procedure. J. Sci. Fd. Agric., 22, 658.

[3] Charney (J.), Fischer (W. F.) and Hegarty (C. P.) (1951). - Manganese as an essential element for sporulation in the genus Bacillus. J. Bact., $62,145$.

[4] EICHNER (K.) (1973). - Indikatoren für beginnende qualitatieve Veränderungen von Lebensmitteln. Dtsche Lebensm.-Rundschau, 69, 4.

[5] FrankLIN (J. G.) (1970). - Spores in milk: problems associated with UHTprocessing. J. Appl. Bact., 33, 180.

[6] Galesloot (T. E.) (1956). - Een eenvoudige metode ter bepaling van het bakteriologisch effekt van sterilisatieprocessen voor melk toegepast op het steriliseren van melk in doorstroom- en flessensterilisatoren. Neth. Milk Dairy J., 10, 77.

[7] KeEney (M.) and BasetTE (R.) (1959). - The determination of hydroxymethylfurfural. J. Dairy Sci., 42, 945. 
[8] Kessler (H. G.) (1981). - Food Engineering and Dairy Technology. Verlag A. Kessler, Freising.

[9] MottaR (J.) et NaudTs (M.) (1979). - La qualité du lait chauffé à ultrahaute température comparée à celle du lait pasteurisé et stérilisé dans la bouteille. Le Lait, 59, 476.

[10] Mottar (J.), Waes (G.), Moermans (R.) and Naudts (M.) (1979). - Sensoric changes in UHT milk during uncooled storage. Milchwiss., 34, 257.

[11] Noomen (A.) (1975). - Proteolytic activity of milk protease in raw and pasteurized cow's milk. Neth. Milk Dairy J., 29, 153.

[12] Waes (G.) (1976). - Aerobic mesophilic spores in raw milk. Milchwiss., 31, 521 .

[13] Williams (D. J.), Franklin (J. G.), Chapman (H. R.) and Clegg (L. F.) (1957). Methods of assessing the sporicidal efficiency of an ultra-high temperature milk sterizing plant. I. Experiments with suspensions of spores in water. J. Appl., 20, 43. 